\title{
Phenomenological Fluids from Interacting Tachyonic Scalar Fields
}

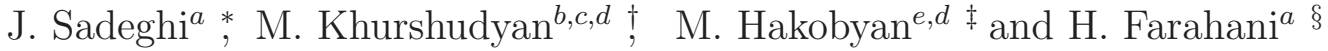 \\ ${ }^{a}$ Department of Physics, Mazandaran University, Babolsar, Iran \\ P.O.Box 47416-95447, Babolsar, Iran \\ ${ }^{b}$ CNR NANO Research Center S3, Via Campi 213a, 41125 Modena MO \\ ${ }^{c}$ Dipartimento di Scienze Fisiche, Informatiche e Matematiche, \\ Universita degli Studi di Modena e Reggio Emilia, Modena, Italy \\ ${ }^{d}$ Department of Theoretical Physics, Yerevan State University, 1 Alex Manookian, 0025, Yerevan, Armenia \\ ${ }^{e}$ A.I. Alikhanyan National Science Laboratory, Alikhanian Brothers St., Yerevan, Armenia \\ ${ }^{d}$ Department of Nuclear Physics, Yerevan State University, Yerevan, Armenia
}

August 15, 2018

\begin{abstract}
In this paper we are interested to consider mathematical ways to obtain different phenomenological fluids from two-component Tachyonic scalar fields. We consider interaction between components and investigate problem numerically. Statefinder diagnostics and validity of the generalized second law of thermodynamics performed and checked. We suppose that our Universe bounded by Hubble horizon.
\end{abstract}

Keywords: FRW Cosmology; Dark Energy; Phenomenology.

\section{Introduction}

Modern cosmology faced with a problem when a set of observational data reveal the following picture of our Universe called modern era in theoretical cosmology. Observations of high redshift type SNIa supernovae [13] reveal the accelerating expansion of our Universe. Then, other series of observations like to investigation of surveys of clusters of galaxies show that the density of matter is very much less than critical density [4], observations of Cosmic Microwave Background (CMB) anisotropy indicate that the Universe is flat and the total energy density is very close to the critical $\Omega_{t o t} \simeq 1$ [5]. Explanation of accelerated expansion of our Universe takes two different ways and now they are developing and evaluating as different approaches, however there is not any natural restriction of a possibilities of recombination of two approaches in one single approach. In that case we believe that joined approach will be more sufficient and rich with new and interesting physics. To explain recent observational data, which reveals accelerated expansion character of the Universe, several models were proposed. One of the possible scenarios (general relativity framework) is the existence of a dark energy with negative pressure and positive energy density giving an acceleration to the expansion [6, 7]. Now, astronomical studies and observational data of WMAP confirm that dark energy occupies about $73 \%$ of the energy of our Universe. Other component, dark matter occupies about $23 \%$, and usual baryonic matter occupies about $4 \%$.

\footnotetext{
*Email: pouriya@ipm.ir

$\dagger$ Email: martiros.khurshudyan@nano.cnr.it

†Email: margarit@mail.yerphi.am

§Email: h.farahani@umz.ac.ir
} 
There are several models to describe dark energy such as the cosmological constant and its generalizations [8], or Chaplygin gas and its generalizations [9-15]. Also there are other candidates for the dark energy which are scalar-field dark energy models. A quintessence field [16] is a scalar field with standard kinetic term, which minimally coupled to gravity. In that case the action has a wrong sign kinetic term and the scalar field is called phantom [17]. Combination of the quintessence and the phantom is known as the quintom model [18]. Extension of kinetic term in Lagrangian yields to a more general frame work on field theoretic dark energy, which is called k-essense [19, 20]. A singular limit of k-essense is called Cuscuton model [21]. This model has an infinite propagating speed for linear perturbations, however causality is still valid. The most general form for a scalar field with second order equation of motion is the Galileon field which also could behaves as dark energy [22].

Among different viewpoints concerning to the nature of the dark component of the Universe, in this article, we accept that it could be described by a scalar field and we choose Tachyon as a scalar field. This paper is organized as follows. After introduction, in the next section, we consider two-component Tachyonic fluid and give motivation of our work. In section 3 we write field equations include an interaction between components. Then check validity of the Generalized Second Law (GSL) of thermodynamics for this setting in section 4. In section 5 the statefinder diagnostics is performed as well. In section 6 we give numerical analysis of the differential equations which obtained in previous sections. Finally in section 7 we give conclusion and discuss about results.

\section{Tachyonic fluid}

Tachyonic fluid described by the following relativistic Lagrangian [23],

$$
L_{T F}=-V(\phi) \sqrt{1-\partial_{i} \phi \partial^{i} \phi} .
$$

Therefore, the stress energy tensor is given by,

$$
T^{i j}=\frac{\partial L_{T F}}{\partial\left(\partial_{i} \phi\right)} \partial^{j} \phi-g^{i j} L_{T F},
$$

which yields to the following energy density and pressure,

$$
\begin{gathered}
\rho=\frac{V(\phi)}{\sqrt{1-\partial_{i} \phi \partial^{i} \phi}}, \\
P=-V(\phi) \sqrt{1-\partial_{i} \phi \partial^{i} \phi},
\end{gathered}
$$

respectively. Our next step is to present the equations (3) and (4) as the following,

$$
\begin{aligned}
\rho & =\rho_{1}+\rho_{2}, \\
P & =P_{1}+P_{2},
\end{aligned}
$$

with the following components,

$$
\begin{aligned}
\rho_{1} & =\frac{V(\phi) \partial_{i} \phi \partial^{i} \phi}{\sqrt{1-\partial_{i} \phi \partial^{i} \phi}}+\alpha F(H), \\
P_{1} & =\beta K(H),
\end{aligned}
$$

and,

$$
\begin{aligned}
& \rho_{2}=V(\phi) \sqrt{1-\partial_{i} \phi \partial^{i} \phi}-\alpha F(H), \\
& P_{2}=-V(\phi) \sqrt{1-\partial_{i} \phi \partial^{i} \phi}-\beta K(H),
\end{aligned}
$$


where $F(H)$ and $K(H)$ are arbitrary function of $H$. Under such splitting we have two-component fluid. First of them describes by EoS (Equation of State) parameter $\omega_{1}$, which is given by,

$$
\omega_{1}=\frac{\beta K(H) \sqrt{1-\partial_{i} \phi \partial^{i} \phi}}{V(\phi) \partial_{i} \phi \partial^{i} \phi+\alpha F(H) \sqrt{1-\partial_{i} \phi \partial^{i} \phi}} .
$$

EoS parameter for the second component reads as,

$$
\omega_{2}=\frac{-V(\phi) \sqrt{1-\partial_{i} \phi \partial^{i} \phi}-\beta K(H)}{V(\phi) \sqrt{1-\partial_{i} \phi \partial^{i} \phi}-\alpha F(H)},
$$

while the total EoS of fluid is

$$
\omega=-\left(1-\partial_{i} \phi \partial^{i} \phi\right) .
$$

The equation (10) reduced to $\omega=-\left(1-\dot{\phi}^{2}\right)$ for spatially homogeneous field. Also remember that $\omega \neq \omega_{1}+\omega_{2}$. The species of components of field are recognize by constants $\alpha$ and $\beta$. If $\alpha$ and $\beta$ are both zero then one component is dust matter with zero EoS and other is the cosmological constant (because of its EoS is $\omega=-1)$.

For simplicity and as a toy model we will assume that $F(H)=K(H)=H^{2}$. Alternative models of dark energy suggest a dynamical form of dark energy, which at least in an effective level, can originate from a variable cosmological constant $[24,25]$, or from various fields, such as a canonical scalar field [26-30] (quintessence), a phantom field [31-38] or the quintom [39-51]. By using some basic of quantum gravitational principles, we can formulate several other models for dark energy and in literature they are known as holographic dark energy paradigm [52-63] and agegraphic dark energy models [64-66].

Motivation to consider such splitting is to connect to the attempts of unification of inflation, dark energy and dark matter appearing in literature, which teach us that we can have fluids with exotic EoS, we can consider modified gravity, which already can be accounted as a base to consider fluids with exotic EoS [67-75]. However, in this stage it is to early to conclude that we found a key approach to the unification problem. At least more deep research should be performed, different types of functions should be considered: from linear to some exotic forms not only function on Hubble parameter, but function of scale factor, Ricci scalar or functions of field and potential. We hope that systematic research in this direction, very soon will give desirable results which we will report systematically. We do not exclude possibility that in some cases obtained results will not have deep connection with Universe. Cosmology itself being interdisciplinary research field and a possibility for testing different results has many open questions, which allowed researchers make some phenomenological assumptions which from first sight have not physical bases, but at the same time we observed that with new experimental data old phenomenological assumptions become as a key ingredient of modern Cosmology and found fundamental physical interpretation.

\section{$3 \quad$ Field equations}

Field equations that govern the dynamics of our Universe in frame work of general relativity read as,

$$
R^{i j}-\frac{1}{2} g^{i j} R_{k}^{k}=T^{i j},
$$

where $T^{i j}$ is given by the equation (2). We consider the flat FRW Universe with the following metric,

$$
d s^{2}=d t^{2}-a(t)^{2}\left(\frac{d r^{2}}{1-k r^{2}}+r^{2} d \theta^{2}+r^{2} \sin ^{2} \theta d \phi^{2}\right) .
$$

In that case the relation (11) gives the following equations,

$$
\left(\frac{\dot{a}}{a}\right)^{2}=\frac{\rho}{3}-\frac{k}{a^{2}},
$$


and,

$$
-\frac{\ddot{a}}{a}=\frac{1}{6}\left(\rho+3 P+\frac{3 k}{a^{2}}\right) .
$$

Also Bianchi identities implying that,

$$
\dot{\rho}+3 H(\rho+P)=0,
$$

where $8 \pi G=c=1$ and $\Lambda=0$ assumed. In this paper we consider flat Universe with $k=0$. The interaction between components formally splits the equation (15) into two equations, with assumptions the transfer of energy from second component to first component, which are given as,

$$
\dot{\rho}_{1}+3 H\left(\rho_{1}+P_{1}\right)=Q,
$$

and

$$
\dot{\rho}_{2}+3 H\left(\rho_{2}+P_{2}\right)=-Q .
$$

We take interaction strength $Q$ as phenomenological approach,

$$
Q=3 b H \rho+\gamma \dot{\rho}
$$

where $b$ and $\gamma$ are constants, bound by observations and $\rho=\rho_{1}+\rho_{2}, \dot{\rho}=\dot{\rho}_{1}+\dot{\rho}_{2}$ and $H=\dot{a} / a$ is Hubble parameter. By using this form of interaction strength, the conservation of energy equations are written as,

$$
(1-\gamma) \dot{\rho}_{1}+3 H\left(1+\omega_{1}-b-b \frac{\rho_{2}}{\rho_{1}}\right) \rho_{1}=\gamma \dot{\rho}_{2},
$$

and

$$
(1+\gamma) \dot{\rho}_{2}+3 H\left(1+\omega_{2}+b+b \frac{\rho_{1}}{\rho_{2}}\right) \rho_{2}=-\gamma \dot{\rho}_{1} .
$$

Questions concerning to interacting dark energies are requiring careful approach, because considered forms and types of interactions between components are of phenomenological character. Different interacting models of dark energy have been investigated. As far as we know no known symmetry in nature prevents or suppresses a non-minimal coupling between dark energy and dark matter. At the same time, no piece of evidence has been so far presented against interactions. It is found that a suitable interaction can help to alleviate the coincidence problem. The forms of interaction term considered in literature very often are of the following forms: $Q=3 H b \rho_{d m}, Q=3 H b \rho_{d e}$, or $Q=3 H b \rho_{t o t}$, where $b$ is a coupling constant and positive $b$ means that dark energy decays into dark matter, while negative $b$ means dark matter decays into dark energy. It was found that the observations may favor the decaying of dark matter into dark energy, while the second law of thermodynamics strongly favors dark energy decays into dark matter. Other forms for interaction term considered in literature are $Q=\gamma \dot{\rho}_{d m}, Q=\gamma \dot{\rho}_{d e}, Q=\gamma \dot{\rho}_{t o t}, Q=3 H b \rho_{i}+\gamma \dot{\rho}_{i}$, where $i=\{d m, d e, t o t\}$. These kind of interactions are either positive or negative and can not change sign. A signchangeable interaction $[76,77]$ could be achieved very simply either considering a possibility of including deceleration parameter,

$$
Q=q(\gamma \dot{\rho}+3 b H \rho),
$$

where $\alpha$ and $\beta$ are dimensionless constants, the energy density $\rho$ could be $\rho_{m}, \rho_{d e}, \rho_{t o t} . q$ is the deceleration parameter given by,

$$
q=-\frac{1}{H^{2}} \frac{\ddot{a}}{a}=-1-\frac{\dot{H}}{H^{2}} .
$$

The term of $\gamma \dot{\rho}$ in $Q$ is introduced from the dimensional point of view. In the next section we investigate GSL of thermodynamics in our system. 


\section{The generalized second law of thermodynamics}

In this section we are going to deal with the question of the validity of the GSL of thermodynamics. For GSL of thermodynamics we will follow Ref. [78], where was considered validity of the GSL of thermodynamics for the Universe bounded by the Hubble horizon 1 ,

$$
R_{H}=\frac{1}{H}
$$

cosmological event horizon,

$$
R_{E}=a \int_{t}^{\infty} \frac{d t}{a}
$$

and the particle horizon,

$$
R_{P}=a \int_{0}^{t} \frac{d t}{a}
$$

The contents in the Universe bounded by the event horizons taken as interacting two components of a single scalar field. The foundation of GSL required the following Gibbs equation of thermodynamics satisfied,

$$
T_{X} d S_{I X}=P d V_{X}+d E_{I X}
$$

where $S_{I X}$ and $E_{I X}=\rho V_{X}$, are internal entropy and energy within the horizon respectively, while $V_{X}=$ $\frac{4}{3} \pi R_{X}^{3}$ denotes the volume of sphere with the horizon radius $R_{A}$. Recall that GSL together with the first law of thermodynamics for the time derivative of total entropy gives,

$$
\dot{S}_{X}+\dot{S}_{I X}=\frac{R_{X}^{2}}{G T_{X}}\left(\frac{k}{a^{2}}-\dot{H}\right) \dot{R}_{X}
$$

while in the case of without the first law we get,

$$
\dot{S}_{X}+\dot{S}_{I X}=\frac{2 \pi R_{X}}{G}\left[R_{X}^{2}\left(\frac{k}{a^{2}}-\dot{H}\right)\left(\dot{R}_{X}-H R_{X}\right)+\dot{R}_{X}\right] .
$$

Under the notations used above we understood that $T_{X}=\frac{1}{2 \pi R_{X}}$ and $R_{X}$ is temperature and radius for a given horizon under equilibrium thermodynamics respectively, $S_{X}$ is the horizon entropy and $\dot{S}_{I X}$ as the rate of change of internal entropy. It was found that the first and second laws of thermodynamics hold on the apparent horizon when the apparent horizon and the event horizon of the Universe are different, while for consideration of only event horizon these laws breakdown [79]. The Friedmann equations and the first law of thermodynamics (on the apparent horizon) are equivalent if the Universe is bounded by the apparent horizon $R_{A}$ with temperature $T_{A}=\frac{1}{2 \pi R_{A}}$ and entropy $S_{A}=\frac{\pi R_{A}}{G}$. Usually, the Universe bounded by apparent horizon and in this region the Bekenstein's entropy-mass bound $\left(S \leq 2 \pi E R_{A}\right)$ and entropy area bound $\left(S \leq \frac{A}{4}\right)$ are hold. Numerical analysis of the equations (27) and (28) given in the section 6 . In order the GSL to be hold it is required that $\dot{S}_{X}+\dot{S}_{I X} \geq 0$ i.e. the sum of entropy of matter enclosed by horizon must be not be a decreasing function of time.

\section{$5 \quad$ Statefinder diagnostics}

In this section we consider problem of statefinder diagnostics. The property of dark energy is model dependent and to differentiate various models of dark energy, a sensitive diagnostic tool is needed. Hubble parameter $H$ and deceleration parameter $q$ are very important quantities which can describe the geometric properties of the Universe. Since $\dot{a}>0$, hence $H>0$ means the expansion of the Universe. Also, $\ddot{a}>0$,

1 Recall that in case when $k=0$ as in our case apparent horizon $R_{A}=\frac{1}{\sqrt{H^{2}+\frac{k}{a^{2}}}}$ we get the radius of the Hubble horizon 
which is $q<0$ indicates the accelerated expansion of the universe. Since, the various dark energy models give $H>0$ and $q<0$, they can not provide enough evidence to differentiate the more accurate cosmological observational data and the more general models of dark energy. For this aim we need higher order of time derivative of scale factor and geometrical tool. Sahni et.al [79] proposed geometrical statefinder diagnostic tool, based on dimensionless parameters $(r, s)$ which are function of scale factor and its time derivative. These are jerk and snap parameters which are define as the following,

$$
\begin{aligned}
r & =\frac{1}{H^{3}} \frac{\dddot{a}}{a}, \\
s & =\frac{r-1}{3\left(q-\frac{1}{2}\right)},
\end{aligned}
$$

respectively. The deceleration parameter is also given by the equation (22). We will use other form of parameters in terms of he total energy density $\rho$ and pressure $P$ in the Universe,

$$
\begin{aligned}
r & =1+\frac{9(\rho+P)}{2 \rho} \frac{\dot{P}}{\dot{\rho}} \\
s & =\frac{(\rho+P)}{P} \frac{\dot{P}}{\dot{\rho}} \\
q & =\frac{1}{2}\left(1+\frac{3 P}{\rho}\right) .
\end{aligned}
$$

The plot of $r, s$ and $q$ are given in the Fig. 1.
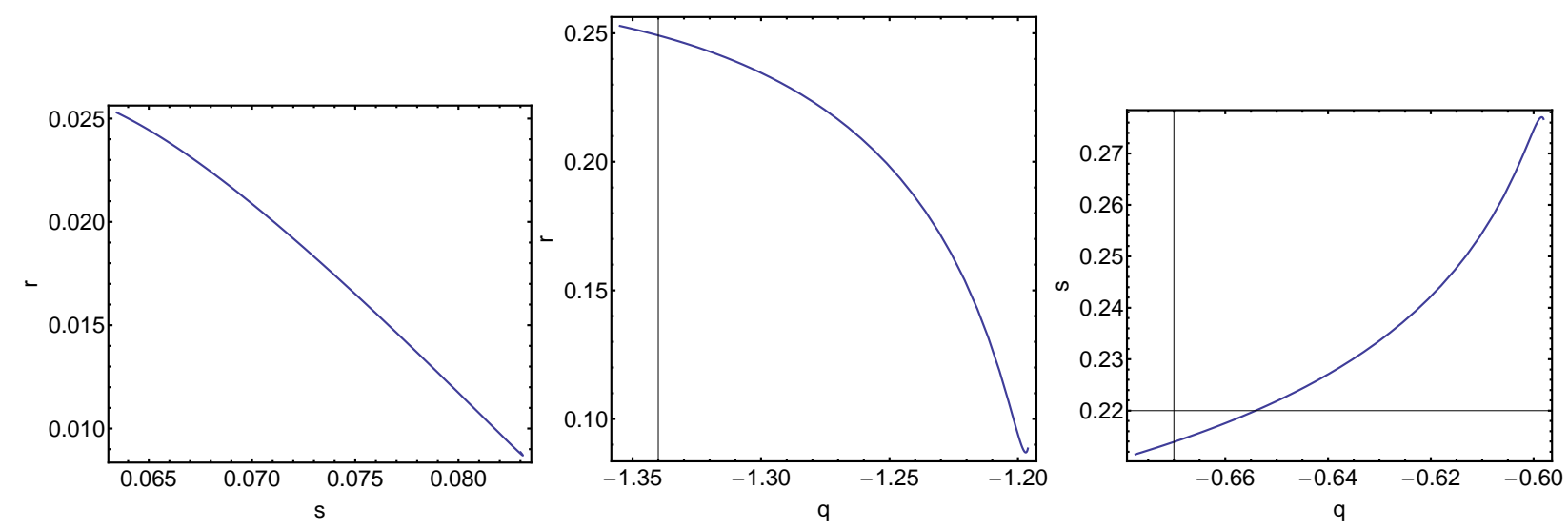

Figure 1: The above plot refer to the links between $r, s$ and $q$. We choose $\alpha=2.5, \beta=1.5, b=0.01$ and $\gamma=0.02$.

\section{Numerical Results}

Numerical analysis provide us following information. First of all we find that field is a complex one and in this case a complex field is able to produce accelerated expansion i.e $q<0$. In the Fig. 2 we present behavior of $\omega_{\text {eff }}=\frac{P_{1}+P_{2}}{\rho_{1}+\rho_{2}}$ of composed fluid, $\omega_{1}, \omega_{2}$ of fluids and $q$ against time $t$ for different values of $\alpha$ and $\beta$ with the fixed valued of interacting constants. We observe that for the composed fluid for early stages of evolution it is a fluid with $\omega>0$ but then we have transition to a dark energy with $\omega>-1$ indicating quintessence-like behavior. We also investigate behavior of $\omega_{1}$ and $\omega_{2}$ of our phenomenological fluids and observe that $\omega_{1}$ is a 

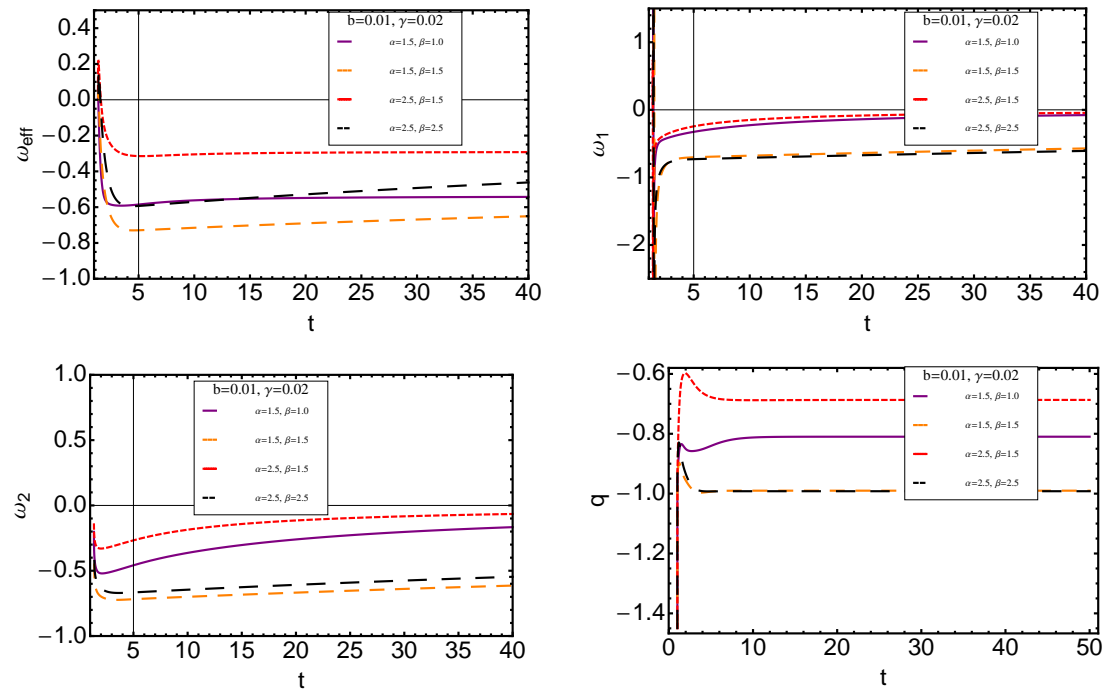

Figure 2: The above plots refer to the links between $\omega_{t o t}, \omega_{1}, \omega_{2}, q$ and $t$ for fixed interaction parameters. In $(q-t)$ plot, it is seen that the Universe initially undergoes a rapidly falling acceleration followed by a rise in it. At a particular epoch the Universe get into a phase of constant acceleration in which we are presently located. We choose $b=0.01$ and $\gamma=0.02$.

positive at early stages and carries fast jump to a dark energy with negative EoS parameter (phantom dark energy) and then during evolution it becomes quintessence with $\omega>-1$. Fast change of the type of the first fluid can have very deep physics. In this letter we assume that, or this behavior related to the fact that Tachyon field is unstable in early stages of evolution, either that first fluid itself is not in thermodynamical equilibrium and some irreversible processes like to a particle creation and annihilation is happening "inside" the fluid. More deep analysis is needed in order to conclude with right physics. On the other hand, we see that $\omega_{2}$ is completely negative for all values of our parameters $(0>\omega>-1)$ which suggest quintessence-like behavior. For the deceleration parameter we see, that it is negative i.e. we get ever accelerated Universe. In the second analysis of the model, for illustration, we fixed values of parameters coming from fluids splitting i.e. $\alpha$ and $\beta$ and investigate behavior of $\operatorname{EoS}$ parameters and $q$ against time for different values of interacting constants (see Fig. 3). We conclude that, for different values of interaction parameters intensively considered in literature discussed from experimental constraints, composed fluid and its components can be identified as the same and first fluid has the same behavior as already discussed above i.e fast transition from a fluid to a dark energy at early stages of evolution. Again, we have ever accelerated Universe.

Validity of the GSL of thermodynamics is satisfied for our model, which can be accounted as a simple and first test telling us about true-like model (see Fig. 4). Other test like comparing the model with experimental data can be also done in future, which is also will allow us to fix a viable range of the values of the model. Finally in the Fig. 5 we present $V(t)$ and $\phi(t)$. We can see that there is a maximum for the potential which is corresponding to the minimum of the $\omega_{e f f}$. Then, Tachyon potential vanished at the late time, which is expected. We find that increasing $\beta$ increased potential while increasing $\alpha$ decreased one. Also we see that $\phi$ is increasing function of time at the late time, while in the early stage it is strongly depend on choosing parameters. We can observe that the parameter $\beta$ decreases value of $\phi$ at the late stage but the parameter $\alpha$ increases one. 

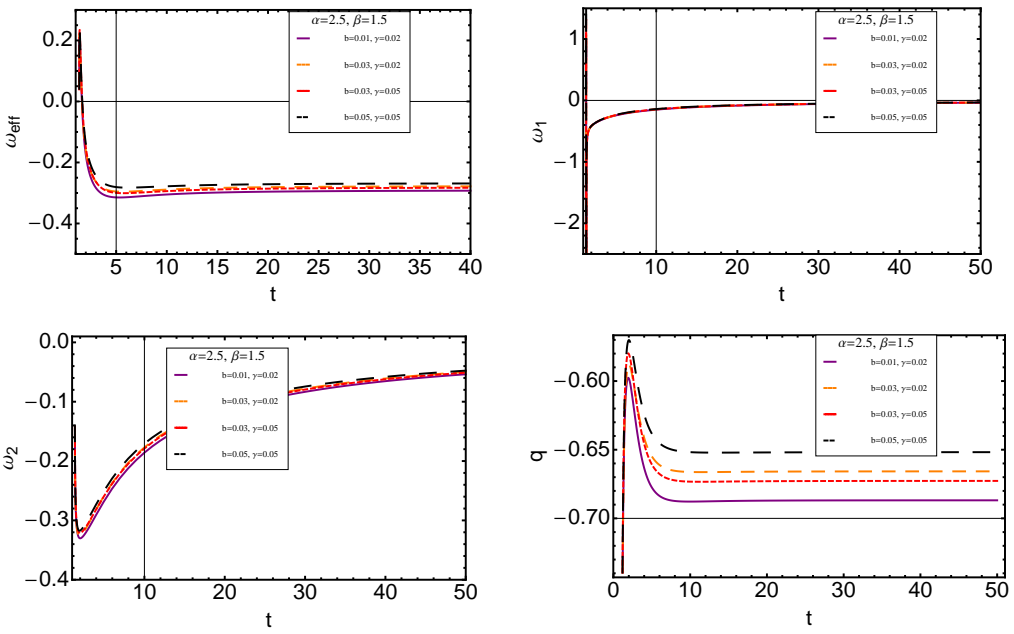

Figure 3: The above plots refer to the links between $\omega_{t o t}, \omega_{1}, \omega_{2}, q$ and $t$ for fixed fluid parameters. In $(q-t)$ plot, it is seen that the Universe initially undergoes a rapidly falling acceleration followed by a rise in it. At a particular epoch the Universe get into a phase of constant acceleration in which we are presently located. We choose $\alpha=2.5$ and $\beta=1.5$.
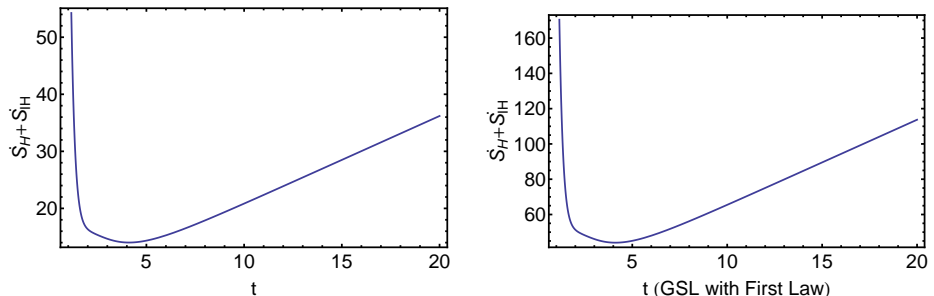

Figure 4: $\dot{S}_{H}+\dot{S}_{I H}$ versus time for the case of, Left: only GSL of thermodynamics (without the first law). Right: GSL together with the first law of thermodynamics. We set $\alpha=2.5, \beta=1.5, b=0.01$ and $\gamma=0.02$.

\section{Conclusion}

In this article we considered a phenomenological splitting of Tachyonic scalar field which gives rise of two fluids. In base of special form of additional term, which is a function of Hubble parameter with an interaction between them we investigated behavior of the Universe. We observed that we have ever accelerated expansion. Fluid responsible for that acceleration for later stages is dark energy with $\omega<0$, while for early stages of evolution it was not dark energy, moreover we have fluid type transition. This behavior can be accepted as counterintuitive related to the fact that, in GR, accelerated expansion caused by dark energy. GR does not give any explanation related to the origin of dark energy as well as about origin of dark meter. Numerical analysis shows that field is a complex and is able to provide discussed acceleration. Validity of the GSL of thermodynamics could be thought as a test giving us a hope, that we could continue our research in this direction. We already mentioned about motivations and about our interests related to the question, which is arose from the research providing different ways for unification of Inflation, dark energy and dark matter, which provides us knowledge that we have a right to consider fluids with exotic EoS like in our case, with one difference that the base of our fluids is Tachyonic field. In future we would like to consider different variations concerning to the forms of functions and parameters and investigate behavior of the Universe and fluid. Also, one can investigate the effects of shear or bulk viscosities on the system. 

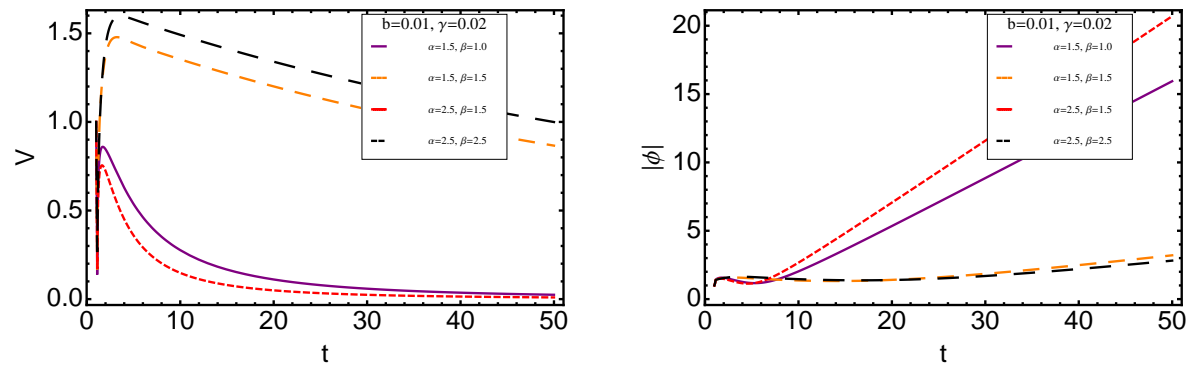

Figure 5: Plots of $V(t)$ and $\phi(t)$, where we choose $b=0.01$ and $\gamma=0.02$.

\section{Acknowledgments}

Martiros Khurshudyan has been supported by EU fonds in the frame of the program FP7-Marie Curie Initial Training Network INDEX NO.289968.

\section{References}

[1] A.G. Riess et al. [Supernova Search Team Colloboration], Astron. J. 116 (1998) 1009

[2] S. Perlmutter et al. [Supernova Cosmology Project Collaboration], Astrophys. J. 517 (1999) 565

[3] R. Amanullah et al., Astrophys. J. 716 (2010) 712

[4] A.C. Pope et al. Astrophys. J. 607 (2004) 655

[5] D.N. Spergel et al. Astrophys. J. Supp. 148 (2003) 175

[6] M. Li, X-D. Li, S. Wang and Y. Wang, "Dark Energy", arXiv:1103.5870 [astro-ph.CO]]

[7] J. Sadeghi, B. Pourhassan, and Z. Abbaspour Moghaddam, "Interacting Entropy-Corrected Holographic Dark Energy and IR Cut-Off Length", Int. J. Theor. Phys. (2013) arXiv:1306.2055 [gr-qc]]

[8] A. Sen, J. High Energy Phys. 0207 (2002) 065 [arxiv: hep-th/0203265

[9] A.R. Amani and B. Pourhassan, "Viscous Generalized Chaplygin gas with Arbitrary $\alpha "$, Int. J. Theor. Phys. 52 (2013) 1309

[10] H. Saadat and B. Pourhassan, "Viscous Varying Generalized Chaplygin Gas with Cosmological Constant and Space Curvature", Int. J. Theor. Phys. DOI: 10.1007/s10773-013-1676-2

[11] H. Saadat and B. Pourhassan, "FRW Bulk Viscous Cosmology with Modified Chaplygin Gas in Flat Space", Astrophysics and Space Science 343 (2013) 783

[12] H. Saadat and B. Pourhassan, "FRW bulk viscous cosmology with modified cosmic Chaplygin gas", Astrophysics and Space Science 344 (2013) 237

[13] B. Pourhassan, "Viscous Modified Cosmic Chaplygin Gas Cosmology" International Journal of Modern Physics D 22 (9) (2013) 1350061 arXiv:1305.6054 [gr-qc]]

[14] J. Sadeghi, M. Khurshudyan, H. Farahani, "Phenomenological Varying Modified Chaplygin Gas with Variable $G$ and $\Lambda$ : Toy Models for Our Universe", arXiv:1308.1819 [gr-qc]] 
[15] M. Khurshudyan, "Interaction between Generalized Varying Chaplygin gas and Tachyonic Fluid", arXiv:1301.1021 [gr-qc]]

[16] C. Wetterich, Nucl. Phys. B 302 (1988) 668

[17] R.R. Caldwell, Phys. Lett. B 545 (2002) 23

[18] B. Feng, X. L. Wang, and X. M. Zhang, Phys. Lett. B 607 (2005) 35

[19] C. Armendariz-Picon, V. F. Mukhanov, and P. J. Steinhardt, Phys. Rev. Lett. 85 (2000) 4438

[20] C. Armendariz-Picon, V. F. Mukhanov, and P. J. Steinhardt, Phys. Rev. D 63 (2001) 103510

[21] N. Afshordi, D. J. H. Chung, and G. Geshnizjani, Phys. Rev. D 75 (2007) 083513

[22] C. Deffayet, et al., arXiv:1103.3260 [hep-th]]

[23] M.M. Verma and S.D. Pathak, "A Thachyonic Scalar Field with Mutually Interacting Components", Int. J. Theor. Phys. 51 (2012) 2370

[24] J. Sola and H. Stefancic, Phys. Lett. B 624 (2005) 147

[25] I.L. Shapiro and J. Sola, Phys. Lett. B 682 (2009) 105

[26] B. Ratra and P.J.E. Peebles, Phys. Rev. D 37 (1988) 3406

[27] I. Zlatev, L. M. Wang and P. J. Steinhardt, Phys. Rev. Lett. 82 (1999) 896

[28] Z. K. Guo, N. Ohta and Y. Z. Zhang, Mod. Phys. Lett. A 22 (2007) 883

[29] S. Dutta, E.N. Saridakis and R.J. Scherrer, Phys. Rev. D 79 (2009) 103005

[30] E.N. Saridakis and S. V. Sushkov, Phys. Rev. D 81 (2010) 083510

[31] R.R. Caldwell, M. Kamionkowski and N.N. Weinberg, Phys. Rev. Lett. 91 (2003) 071301

[32] S. Nojiri and S. D. Odintsov, Phys. Lett. B 562 (2003) 147

[33] P. Singh, M. Sami and N. Dadhich, Phys. Rev. D 68 (2003) 023522

[34] J.M. Cline, S. Jeon and G.D. Moore, Phys. Rev. D 70 (2004) 043543

[35] V.K. Onemli and R.P. Woodard, Phys. Rev. D 70 (2004) 107301

[36] W. Hu, Phys. Rev. D 71 (2005) 047301

[37] M.R. Setare and E. N. Saridakis, JCAP 0903 (2009) 002

[38] E.N. Saridakis, Nucl. Phys. B 819 (2009) 116

[39] E. Elizalde, S. Nojiri and S.D. Odintsov, Phys. Rev. D 70 (2004) 043539

[40] Z.K. Guo, et al., Phys. Lett. B 608 (2005) 177

[41] M.-Z Li, B. Feng, X.-M Zhang, JCAP, 0512 (2005) 002

[42] B. Feng, M. Li, Y.-S. Piao and X. Zhang, Phys. Lett. B 634 (2006) 101

[43] S. Capozziello, S. Nojiri and S.D. Odintsov, Phys. Lett. B 632 (2006) 597

[44] W. Zhao and Y. Zhang, Phys. Rev. D 73 (2006) 123509

[45] Y.F. Cai, T. Qiu, Y.S. Piao, M. Li and X. Zhang, JHEP 0710 (2007) 071 
[46] E.N. Saridakis and J.M. Weller, Phys. Rev. D 81 (2010) 123523

[47] Y.F. Cai, T. Qiu, R. Brandenberger, Y.S. Piao and X. Zhang, JCAP 0803 (2008) 013

[48] M.R. Setare and E.N. Saridakis, Phys. Lett. B 668 (2008) 177

[49] M.R. Setare and E.N. Saridakis, Int. J. Mod. Phys. D 18 (2009) 549

[50] Y.F. Cai, E.N. Saridakis, M.R. Setare and J.Q. Xia, Phys. Rept. 493 (2010) 1

[51] T. Qiu, Mod. Phys. Lett. A 25 (2010) 909

[52] S.D.H. Hsu, Phys. Lett. B 594 (2004) 13

[53] M. Li, Phys. Lett. B 603 (2004) 1

[54] Q.G. Huang and M. Li, JCAP 0408 (2004) 013

[55] M. Ito, Europhys. Lett. 71 (2005) 712

[56] X. Zhang and F.Q. Wu, Phys. Rev. D 72 (2005) 043524

[57] D. Pavon and W. Zimdahl, Phys. Lett. B 628 (2005) 206

[58] S. Nojiri and S.D. Odintsov, Gen. Rel. Grav. 38 (2006) 1285

[59] E. Elizalde, S. Nojiri, S.D. Odintsov and P. Wang, Phys. Rev. D 71 (2005) 103504

[60] H. Li, Z.K. Guo and Y.Z. Zhang, Int. J. Mod. Phys. D 15 (2006) 869

[61] E.N. Saridakis, Phys. Lett. B 660 (2008) 138

[62] E.N. Saridakis, JCAP 0804 (2008) 020

[63] E.N. Saridakis, Phys. Lett. B 661 (2008) 335

[64] R.G. Cai, Phys. Lett. B 657 (2007) 228

[65] H. Wei and R.G. Cai, Phys. Lett. B 660 (2008) 113

[66] H. Wei and R.G. Cai, Eur. Phys. J. C 59 (2009) 99

[67] S. Nojiri, S.D. Odintsov, "Introduction to modified Gravity and gravitational alternative for dark energy", Int. J. Geom. Meth. Mod. Phys. 4 (2007) 115 arXiv:hep-th/0601213

[68] S. Nojiri, S.D. Odintsov, "Modified gravity and its reconstruction from the universe expansion history", J. Phys. Conf. Ser. 66 (2007) 012005 arXiv:hep-th/0611071

[69] M.M. Ivanov, A. V. Toporensky, "Stable super-inflating cosmological solutions in $f(R)$ gravity", Int. J. Mod. Phys. D 21 (2012) 1250051 arXiv:1112.4194 [gr-qc]]

[70] K. Bamba, S.D. Odintsov, "Lorenzo Sebastiani and Sergio Zerbini, Finite-time future singularities in modified Gauss-Bonnet and $F(R, G)$ gravity and singularity avoidance", arXiv:0911.4390 [hep-th]]

[71] S. Nojiri, S.D. Odintsov, "The future evolution and finite-time singularities in $F(R)$-gravity unifying the inflation and cosmic acceleration", Phys. Rev. D78 (2008) 046006 arXiv:0804.3519 [hep-th]]

[72] Y-F. Cai, S-H. Chen, J.B. Dent, S. Dutta and E.N. Saridakis, "Matter Bounce Cosmology with the $f(T)$ Gravity", Class. Quantum Grav. 28 (2011) 215011 [arXiv:1104.4349 v]

[73] C-H. Chuang, J-A. Gu, and W-Y.P. Hwang, "Inhomogeneity-Induced Cosmic Acceleration in a Dust Universe", Class. Quant. Grav. 25 (2008) 175001 arXiv:astro-ph/0512651 
[74] W. Hao, "Cosmological Constraints on the Sign-Changeable Interactions", Common. Theory. Phys. 56 (2011) 972

[75] H. Wei, Nucl. Phys. B 845 (2011) 381

[76] A. Das, S. Chattopadhyay, U. Debnath, "Validity of the Generalized Second Law of Thermodynamics in the Logamediate and Intermediate Scenarios of the Universe", Found. Phys. 42 (2012) 266

[77] B. Wang, Y. G. Gong, E. Abdalla.: Phys. Rev. D 74 (2006) 083520

[78] R.G. Cai, L.M. Cao Phys. Rev. D 75 (2007) 064008

[79] V. Sahni, T.D. Saini, A.A. Starobinsky, and U. Alam, "Statefinder - a new geometrical diagnostic of dark energy", JETP Lett. 77 (2003) 201 